\title{
La traduction peut-elle abattre les frontières linguistiques et culturelles?
}

\author{
Agatino LO CASTRO \\ Université Paris-Est Créteil, France \\ Université de Catane, Italie
}

Résumé : La traduction représente un acte de paix et d'échange qui vise à dépasser les frontières linguistiques et culturelles. En effet, elles sont à repenser au prisme d'une réflexion sur la traduction en tant que pratique transculturelle visant à l'acceptation de l'autre et aux rencontres des langues et des cultures. Plusieurs questions en découlent : Quel type de réflexion sur la traduction peut-on envisager pour abattre les frontières linguistiques et culturelles ? Faut-t-il penser la traduction comme un acte uniquement linguistique ? Est-il possible d'accepter l'autre d'un point de vue linguistique et culturel pour concevoir la traduction comme un acte transculturel ? Nous essayerons de répondre à ces questions en analysant et en traduisant en italien des extraits du recueil poétique Prière aux ancêtres du poète franco-congolais Gabriel Okoundji.

\begin{abstract}
Translation is an act of peace and exchange that aims to transcend linguistic and cultural boundaries. Indeed, translation is a transcultural practice that aims at the acceptance of the other and the encounters of languages and cultures. Several questions arise from this: What type of reflection on translation can be envisaged to break down linguistic and cultural boundaries? Should we think of translation as a purely linguistic act? Is it possible to accept the other from a linguistic and cultural point of view to conceive translation as a transcultural act? We will try to answer these questions by analyzing and by translating extracts into Italian from Prière aux ancêtres by the FrenchCongolese poet Gabriel Okoundji.
\end{abstract}

Mots clés : transculturalité, traductologie, frontière, poésie, Gabriel Okoundji

Keywords: transcultural studies, translation, border, poetry, Gabriel Okoundji

\section{Introduction}

La traduction représente un acte de paix, de rencontre entre les cultures, les langues et les expériences humaines. Elle devient un acte à la fois linguistique et culturel qui vise à l'acceptation de l'autre, voire à l'interaction des langues et des cultures, dans une perspective transculturelle, c'est -à dire « un ensemble de transmutations constantes des éléments des cultures en présence : en même temps qu'on donne un élément, on en reçoit un autre de sorte qu'à la fin de l'opération, telle une équation chimique une réalité nouvelle en émerge indépendante des 


\section{sciendo}

modèles de base » (Semujanga, 2005: 180). Cette approche est possible si l'on accepte l'autre en traduction et si l'on vise à mettre l'accent sur les diversités culturelles et linguistiques.

En ce qui concerne la réflexion sur la traduction, accepter et intégrer la diversité des langues et des cultures pourrait mettre l'accent sur la traduction en tant que pratique pour dépasser les frontières linguistiques et culturelles. Par rapport à la pratique traductive, il serait indispensable de se pencher sur la biographie linguistique des auteur.e.s et sur les dynamiques transculturelles et translinguistiques des textes. Plusieurs questions découlent : Quel type de réflexion sur la traduction peut-on envisager pour abattre les frontières linguistiques et culturelles ? Faut-t-il penser la traduction comme un acte uniquement linguistique ? Est-il possible d'accepter l'autre d'un point de vue linguistique et culturel pour concevoir la traduction comme un acte transculturel visant au dépassement des frontières?

Dans la première partie, nous analysons le concept de frontière et de nation d'après les définitions proposées par le Trésor de la langue française informatisé, ce qui nous aide à orienter notre réflexion; dans la deuxième partie nous mettons l'accent sur l'idée d'hospitalité et d'acceptation de l'autre en traduction, ce qui nous permet d'envisager le dépassement des frontières linguistiques et culturelles sur le plan théorique; dans la troisième partie, nous proposons la traduction en italien de deux extraits tirés du recueil poétique Prière aux ancêtres du poète franco-congolais Gabriel Okoundji (Okoundji \& Tardif 2007), ce qui nous permet(i) d'abord de réfléchir sur le rapport français-tégué ( les langues du poète), (ii) ensuite d'accepter les éléments linguistiques et culturels en traduction italienne (iii) et de valoriser l'aspect transculturel de la traduction, afin de dépasser les frontières linguistiques et culturelles.

\section{Pour une réflexion sur les définitions de frontière et de nation}

Avant de réfléchir sur la notion de frontière, nous proposons d'analyser la définition de frontière, telle qu'elle paraît dans le TLFi :

FRONTIÈRE, subst. fém.

A. - Limite qui, naturellement, détermine l'étendue d'un territoire ou qui, par convention, sépare deux États :

B.- Au fig. Limite, point de séparation entre deux choses différentes ou opposées. (TLFi)

D'après cette définition, nous constatons que (i) le concept de frontière fait référence à la séparation géographique des territoires et (ii) que l'aspect figuré souligne la séparation entre des éléments différents. 
La frontière délimite deux éléments qui ne se connaissent pas. Elle détermine la séparation géographique d'un territoire qui sépare un Etat ou encore elle désigne la ligne de séparation entre deux éléments différents et des pays différents. À cet égard, quel est son rapport avec la nation ? Nous analysons ensemble la définition de nation, telle qu'elle paraît dans le TLFi :

\section{NATION}

B. 1. a) Groupe humain, généralement assez vaste, dont les membres sont liés par des affinités tenant à un ensemble d'éléments communs ethniques, sociaux (langue, religion, etc.) et subjectifs (traditions historiques, culturelles, etc.) dont la cohérence repose sur une aspiration à former ou à maintenir une communauté.

2. ... l'idée même de nation en général ne se laisse pas capturer aisément (...). Le fait essentiel qui les constitue [les nations], leur principe d'existence, le lien interne qui enchaîne entre eux les individus d'un peuple, et les générations entre elles, n'est pas, dans diverses nations de la même nature. (TLFi)

D'après la définition de nation, les éléments d'affinité pris en considération sont la langue, la religion, le territoire. Il est donc possible de voir que les idées d'échange transculturel et de diversité culturelle ne sont pas fortement accentuées. En effet, il est très important de mobiliser deux perspectives différentes de nation. D'une part une conception ethnique de la nation, c'est-à-dire "la nation comme identité [...] fermée, constituée par des liens naturels, qui se sont réalisés dans une communauté de personnes unies par la même langue, la même culture et la même religion » (Tardivel, 2016:54) et d'autre part une conception ouverte de la nation, c'est-à-dire "constituée par des liens contractuels, qui se sont réalisés historiquement dans une communauté de personnes unies par le désir de vivre ensemble sous les mêmes lois, les mêmes mœurs et les mêmes institutions » (Tardivel, $2016: 53-54)$. Toutefois, nous constatons que même dans la conception ouverte de la nation, l'idée d'échange transculturel et de diversité ne sont pas accentuées. Nous n'avons pas ici la présomption de conduire une réflexion géopolitique et historique sur les notions de frontière et de nation, mais nous les interrogeons au prisme des réflexions sur la traduction, pour concevoir le dépassement des frontières culturelles et linguistiques dans la pratique traductive. Par ailleurs, il nous semble assez révélatrice la notion de imagined communities proposée par Benedict Anderson :

"It is imagined because the members of even the smallest nation will never know most of their fellow-members, meet them, [...], yet in the minds of each lives the image of their communion. [...] The nation is imagined as limited because even the largest of them, encompassing perhaps a billion living human beings, has finite, if elastic, boundaries, beyond which lie other nations. No nation imagines itself coterminous with mankind. [...] it is imagined as a community, because, regardless of 
the actual inequality and exploitation that may prevail in each, the nation is always conceived as a deep, horizontal comradeship. " (Anderson, 1991: 6-7).

D'après cette notion, la nation devient imagée et abstraite, surtout si l'on fait référence au débat autour de la transculturalité et la relativité des frontières car :

" [elles sont] construites pour délimiter des États, séparer des entités territoriales, protéger des populations d'autres groupes, les limites internationales sont en train de se transformer en profondeur, tant dans leurs formes que dans leurs fonctions. Ces changements ont déjà commencé à bouleverser nos vies, notre façon de nous ancrer dans les territoires, nos capacités à voyager, mais aussi la définition de nos relations politiques. » (Amilhat Szary, 2015:8)

Il s'agit donc d'une séparation politique qui n'est pas forcément en lien avec l'ancrage culturel et linguistique des dynamiques transculturelles propres à la traduction et aux expériences collectives qui visent à mettre l'accent sur la pluralité culturelle et linguistique. En effet, la frontière pourrait devenir un lieu d'échange et de rencontre si l'on dépasse l'enjeu uniquement politique, pour rejoindre l'aspect humain qui vise à souligner l'importance des expériences humaines, car «habiter la frontière évoque la relation. Elle dit que les peuples se sont rencontrés, quelques fois dans la violence, la haine, le mépris, et qu'en dépit de cela, ils ont enfanté du sens» (Miano, 2012 : 11). Le rapport avec l'autre s'instaure dans une perspective de débat, de relation, d'écoute, de respect et d'équilibre culturel et linguistique. Il s'agit d'une idée de frontière qui dépasse l'enjeu uniquement politique pour rejoindre l'aspect individuel. Dans ce contexte, la langue joue un rôle fondamental, car elle devient le noyau central pour repenser le concept de frontière en traduction. Or compte tenu de ces réflexions, aussi bien la notion de frontière que de nation doivent être repensées au sein de la pratique traductive, c'est-à-dire en envisageant une approche transculturelle de la traduction visant au dépassement des barrières linguistiques et culturelles.

\section{La traduction comme étape pour dépasser les frontières}

Après avoir analysés les définitions de nation et de frontière, il très important de réfléchir sur le rôle du traducteur, sur les rencontres entre les cultures et sur l'importance d'accueillir l'autre en traduction, tout en mettant l'accent sur les dynamiques transculturelles. Les langues représentent l'expression des cultures et des interactions transculturelles et 
translinguistiques. C'est pourquoi, il serait nécessaire d'effacer les frontières linguistiques et culturelles, tout en acceptant l'autre, c'est-àdire reconnaitre les différences et les concevoir dans une perspective transculturelle, car la traduction est un moment de rencontre où les cultures et les langues entrent en contact :

«la traduction étant le plus souvent représentée comme une communication entre les cultures ; information, et seul moyen d'accéder à ce qui est énoncé dans d'autres langues, [...]. Le traducteur est représenté comme un passeur. On ne voit pas, il me semble, qu'on retire par là toute sa spécifité littéraire. [...]. Passeur est une métaphore complaisante. Ce qui importe n'est pas de faire passer. Mais dans quel état arrive ce qu'on a transporté de l'autre côté. » (Meschonnic, 1999: 19)

Or le traducteur est chargé d'un devoir à la fois complexe et fascinant, car il propose et encourage les rencontres entre des cultures et des langues, en cherchant de respecter et d'enrichir les langues et les cultures selon le cas. Chaque langue est unique, dans sa particularité qui se traduit dans l'acceptation des dynamiques transculturelles. En effet, traduire devient un défi difficile, mais très stimulant, car il s'agit de concevoir la traduction comme un lieu de rencontre, où l'on doit avoir le courage d'introduire l'étranger et les aspects créatifs et culturels de ce choix, ce qui permet d'abattre les frontières linguistiques et culturelles. En résumant, le traducteur doit :

"savoir écouter la mélodie et les harmonies, repérer la rythmique et toute l'organisation en système des sonorités reviendrait à être à l'écoute de l'Autre, de son discours, d'entendre son identité Unique et originale qui viendra. » (Raguet, 2007:1)

\subsection{L'hospitalité et l'acceptation}

Dans la pratique de la traduction, le texte, le lecteur, l'auteur et le traducteur entrent en contact, car «[la]poetica dell'ospitalità, [...] si configura altresì come un rapporto dialogico tra il traduttore e le fonti, teoriche e letterarie, che hanno maggiormente influenzato la sua pratica».1 (Casini, 2014: 2). Dans cette perspective, le concept d'hospitalité en traduction fait ressortir un rapport de respect entre les langues et les cultures, car :

1 «[la]poétique de l'hospitalité, [...] se présente également comme une relation dialogique entre le traducteur et les sources, théoriques et littéraires, qui ont le plus influencé sa pratique » (notre traduction). 
« l'ospitalità è l'esperienza di una cultura che riconosce l'altro senza sottrarre all'altro la sua alterità o diversità, la sua identità di carattere e sapere e costume, e nello stesso tempo pone colui che ospita nella condizione di non dover rinunciare alla sua singolarità, alla sua identità. »11(Prete, 2011: 16)

L'hospitalité aboutit à l'acceptation de l'autre dans le texte traduit, dans une perspective transculturelle de la traduction. L'expérience traductive, les rapports entre les sociétés et les cultures se voient forcément transculturels, car il y a un équilibre où l'on donne et l'on reçoit, dans la perspective d'un enrichissement culturel et linguistique, car :

«Die meisten unter uns sind in ihrer kulturellen Formation durch mehrere kulturellen Herkünfte und Verbindungen bestimmt. Wir sind kulturelle Mischlinge. Die kulturelle Identität der heutigen Individuen ist eine patchworkIdentität. [...] Das betrifft nicht etwa nur Migranten und Migrantinnen, sondern alle Heranwachsenden. [...] Heutige Menschen werden zunehmend in sich transkulturell ». »2(Welsch, 2010 : 45-46)

Cette approche vise à mettre l'accent sur l'expérience humaine, car elle permet de dépasser les frontières des cultures et de les interpréter au prisme d'une réflexion sur la traduction. Elles deviennent des lieux de rencontre et d'enrichissement personnel, où l'expérience transculturelle permet cet échange. En effet:

« en reprenant le terme de « zone » comme pierre de touche théorique, j'ai voulu imaginer une vaste topographie intellectuelle qui ne soit ni la propriété d'une seule nation, ni une aire aux frontières floues associée au postnationalisme, mais une zone d'engagement critique qui mette en rapport le l et le $\mathrm{n}$ de transLation et transNation. » (Apter, 2015 : 1213)

C'est donc à travers cette conception transnationale et transculturelle que l'on peut envisager la coopération entre les

\footnotetext{
${ }^{1}$ « l'hospitalité est l'expérience d'une culture qui reconnaît l'autre sans soustraire à l'autre son altérité ou sa diversité, son identité de caractère, de savoir et de coutume, et en même temps met celui qui accueille dans la condition de ne pas devoir renoncer à sa singularité, à son identité » ( notre traduction).

${ }^{2}$ « La plupart d'entre nous, dans leur formation culturelle, sont déterminés par plusieurs origines et liens culturels. L'identité culturelle des individus d'aujourd'hui est une identité patchwork. [...] Cela ne concerne pas seulement les migrants, mais tous les adolescents. [...] Les gens d'aujourd'hui sont de plus en plus transculturels » (notre traduction).
} 
langues et les cultures et le dépassement des notions de nation et frontière par le biais de la pratique traductive.

\subsection{Traduire l'autre : l'exemple de Prière aux ancêtres}

Pour répondre aux questions que nous avons posé dans l'introduction à cet article et pour voir dans quelle mesure la traduction peut abattre les frontières linguistiques et culturelles dans la pratique traductive, nous avons choisi d'analyser deux extraits du recueil poétique Prière aux ancêtres du poète franco-congolais Gabriel Okoundji. Avant de nous lancer dans l'expérience traductive, nous prenons en examen la biographie linguistique de l'auteur, ce qui nous permet de remarquer les particularités liées aux langues d'expression du poète et d'en comprendre les spécificités linguistiques et culturelles. Nous reprenons les mots de l'auteur :

«La langue Tégué est donc tout naturellement ma langue parentale; et le français ma langue d'écriture. Entre ces deux langues, j'avoue aujourd'hui ne plus savoir reconnaitre exactement la part de l'affluent et celle du confluent. Ces deux langues coulent en moi ; elles forment harmonieusement ce j'ai coutume d'appeler «l'unité de ma langue maternelle» : Celle qui me permet de nommer les bruits du cœur avec les mots du cœur. Quand l'une d'elle invoque, l'autre évoque, quand l'une donne, l'autre reçoit, et vice versa. Ma quête poétique réside fondamentalement dans cet équilibre. » (Okoundi et Quiller 2009,42)

Le français et le tégué cohabitent et se mélangent dans l'expression écrite. Pour l'auteur, l'impossibilité de les distinguer s'exprime dans un plurilinguisme culturel et dans les dynamiques transculturelles du texte. Il est donc très important d'accepter les éléments aussi bien linguistiques que culturels dans la traduction en langue italienne, ce qui permet de dépasser les notions de frontière et de nation au prisme d'une vision transculturelle de la traduction.

\subsection{Proposition de traduction}

Dans les textes de Gabriel Okoundji, la langue française -langue d'écriture du poète- cohabite avec la culture tégué, ce qui devient l'élément à retenir si l'on veut abattre les frontières culturelles et accepter l'autre, car il s'agit d'un moment de rencontre entre les cultures et les langues en question. Si d'un côté, la langue tégué est la langue de la mémoire, de l'autre, la langue française filtre les éléments de la culture tégué. Dans quelle manière est-il possible de reprendre les spécificités du texte okoundjien en italien? Nous examinons cet extrait : 


\begin{tabular}{|c|c|}
\hline $\begin{array}{l}\text { Majestueuse terre de Mpana aux } \\
\text { vaisseaux chargés de douleur }\end{array}$ & $\begin{array}{l}\text { Maestosa terra di Mpana carica di } \\
\text { barche di dolore }\end{array}$ \\
\hline $\begin{array}{l}\text { terre née de la blessure profonde } \\
\text { d'un cour trahi }\end{array}$ & $\begin{array}{l}\text { terra nata dalla ferita profonda di } \\
\text { un cuore tradito }\end{array}$ \\
\hline $\begin{array}{l}\text { terre de cendres, territoire } \\
\text { d'immenses éclairs }\end{array}$ & $\begin{array}{l}\text { terre di ceneri, territorio } \\
\text { d'innumerevoli lampi }\end{array}$ \\
\hline $\begin{array}{l}\text { qu'a-t-on fait du souffle Tégué de } \\
\text { tes maîtres féticheurs? }\end{array}$ & $\begin{array}{l}\text { cosa ne hai fatto del soffio Tégué dei } \\
\text { tuoi maestri fattucchieri? }\end{array}$ \\
\hline $\begin{array}{l}\text { Terre de malheur, la misère de } \\
\text { l'Homme est aveugle }\end{array}$ & $\begin{array}{l}\text { Terra di sfortuna, la miseria } \\
\text { dell'Uomo è cieca }\end{array}$ \\
\hline $\begin{array}{l}\text { les Mwènè annoncent la destinée } \\
\text { au tréfonds des âmes }\end{array}$ & $\begin{array}{l}\text { i Mwènè annunciano il destino nella } \\
\text { profondità dell'anima }\end{array}$ \\
\hline $\begin{array}{l}\text { la destinée toute silencieuse est à } \\
\text { l'ombre des bruits de la nuits }\end{array}$ & $\begin{array}{l}\text { il destino completamente muto è } \\
\text { all'ombra dei rumori della notte }\end{array}$ \\
\hline $\begin{array}{l}\text { mais voilà que la savane a perdu } \\
\text { le visage de la panthère (p. } 39)\end{array}$ & $\begin{array}{l}\text { ma ecco che la savana ha perso il } \\
\text { viso della pantera (notre } \\
\text { traduction) }\end{array}$ \\
\hline
\end{tabular}

Nous avons gardé les images, les métaphores et les figures issues de la langue tégué en langue italienne, ce qui permet la rencontre culturelle entre la culture tégué d'expression française et la langue italienne. Nous avons gardé l'obscurité énigmatique du texte okoundjien même en langue italienne, car des figures comme panthère (pantera) ont une particularité propre au texte. En particulier, le rôle de la panthère est très important dans la culture tégué, car « en langue Téké et Tégué [...] ko ou kwa signifie: lieu; et ngo ou ngoh, la panthère. En langue kongo, kwango signifie le pays de la panthère » (Chevrier, 2014: 51). Il s'agit donc d'un symbole cher au monde poétique du poète. Nous avons gardé aussi le lexème /Mwènè/ en italien qui est propre à la culture tégué, ce qui devient un emprunt à la langue tégué et un lexème en langue italienne. En effet, ce lexème n'existe pas en italien, il est donc notre préoccupation de le garder et d'expliquer au lecteur la valeur de cette figure en écrivant une note du traducteur. En effet, "le Mwènè est le Nkani, [...]; on l'appelle : Okoundji, le Mokoundji, garant du pouvoir traditionnel moral et judiciaire, il est celui qui assure le lien entre les mondes du visible et de l'invisible. » (Okoundji, 2009 : 49).

Or il est donc très important d'effectuer un travail de recherche avant de se lancer dans la pratique traductive. Il serait donc impossible de se limiter à une austère traduction des signes linguistiques, sans avoir analysé le contexte culturel, les rôles des langues dans le texte et les dynamiques internes aux textes. Dans le cadre de la traduction de cet extrait, nous avons vu que (i) d'une part la panthère, (ii) d'autre part le Mwènè sont des figures et des symboles assez révélateurs dans la poétique okoundjienne. C'est pourquoi, il est notre prérogative de les accueillir dans la traduction italienne, voire d'abattre les frontières 
culturelles et linguistiques. Prenons pour exemple, Majesteuse terre, que nous avons traduit en italien par Maestosa terra. Nous avons préservé ainsi l'ordre syntaxique du français (adjectif+ substantif), parce que dans l'unité à traduire du français, nous identifions une structure presque " rituelle », liée à la liturgie d'un hymne à l'initiation. Par ailleurs, nous avons pu remarquer que le choix de maintenir la même structure en langue italienne (adjectif+ substantif) nous a facilité le respect et le transfert de l'idée de ritualité, voire d'un rite d'initiation. C'est ainsi que nous avons pu faire comprendre aux lecteurs italiens cet effet. Il s'agit (i) d'abord de respecter les dynamiques internes au texte, (ii) ensuite de reproduire cette dynamique en italien en gardant la structure adjective+substantif (Maestosa terra) qui reprend la structure française (Majeusteuse terre) et la dimension " rituelle » que nous avons évoquée. Le choix de garder cette structure renvoi à la volonté d'introduire en italien cette idée de ritualité, ce qui est une prérogative des textes de Gabriel Okoundji.

Or nous avons traduit le lexème français /territoire/ avec le lexème italien /territorio/, vu que le rapprochement phonétique entre les lexèmes italien et français facilite la préservation d'une certaine oralité élément qui prévaut dans l'œuvre de l'auteur. Le lexème /territoire/ évoque l'attachement physique à la /terre/ (terra, en italien), en faisant écho la répétition du lexème /terre/ dans l'extrait pris en examen que nous avons traduit en italien avec /terra/. Nous avons donc décidé de garder ce lien entre terre et territoire (en it. terra et territorio).

Ensuite, il est très important de remarquer l'importance des formules de sagesse énigmatiques qui doivent être gardées en italien, si l'on veut respecter les dynamiques du texte. Prenons comme exemple l'expression française "mais voilà que la savane a perdu le visage de la panthère ». Nous avons traduit en italien " ma ecco che la savana ha perso il viso della pantera » qui permet de garder l'énigmaticité de cette expression en italien. Le traducteur ne doit pas expliciter le texte, mais lui rendre justice en respectant la valeur des rencontres culturelles. En effet, on peut envisager d'expliquer ces éléments dans une note du traducteur, comme dans le cas précédent, ce qui permet d'orienter le lecteur italien dans l'univers poétique de Gabriel Okoundji.

Prenons en examen l'extrait suivant : VII

[...]

nos cœurs en haillons parmi les cœurs égarés danseront dans le feu de la vie

nos cœurs danseront dans la fougue de la tristesse sur les chemins de la douleur (p. 43)

\section{VII}

[...]

i nostri cuori in stracci tra i cuori smarriti danzeranno nel fuoco della vita

i nostri cuori danzeranno nella foga della tristezza sui cammini del dolore (notre traduction) 
Nous avons traduit l'expression française " nos cœurs en haillons parmi les cœurs égarés danseront dans le feu de la vie » avec «i nostri cuori in stracci tra i cuori smarriti danseranno nel fuoco della vita». Dans la traduction en langue italienne, nous avons gardé la même image que le texte français propose, c'est-à-dire " cuori in stracci ». Nous avons donc introduit une image du texte okoundjien en langue italienne "cuori a stracci». Il s'agit d'un choix bien précis, chercher à introduire en langue italienne une image issue de la culture tégué du poète. Encore une fois, nous n'avons pas expliqué le texte, mais nous avons respecté l'esprit et nous avons renforcé la rencontre entre deux cultures différentes. Or nous avons traduit l'expression française «nos cœurs danseront dans la fougue de la tristesse sur les chemins de la douleur» avec «i nostri cuori danseranno nella foga della tristezza sui cammini del dolore ». Nous remarquons la présence de la personnification du cœur qui est animé dans le texte par rapport à ce que l'on peut trouver dans les définitions des dictionnaires. Cette expression traduit l'intention de remarquer l'importance des formules de sagesse et énigmatiques dans l'œuvre de Gabriel Okoundji. Notre intérêt est de transmettre aux lecteurs italiens les messages, la profondeur linguistique et culturelle des textes, ce qui nous permet d'abattre les frontières linguistiques et culturelles. Il est très important de mettre l'accent sur l'importance de la diversité culturelle pour permettre l'effacement des frontières. C'est donc au traducteur d'accepter l'autre, de se libérer des structures idéologiques qui amèneraient à une traduction ethnocentrique.

\section{Conclusion}

L'étude des vocables frontière et nation, selon les définitions comprises dans le Trésor de la langue française informatisé nous a permis de cadrer notre recherche et de mettre l'accent sur le dépassement des frontières culturelles et linguistiques au prisme d'une réflexion sur la traduction en tant que pratique transculturelle. Le fait d'avoir renvoyé aux concepts d'hospitalité et d'acceptation de l'autre au prisme de la traduction, nous a facilité la compréhension de la manière de franchir les frontières culturelles sur le plan théorique. Les traductions que nous évoquées ont permis de tester nos hypothèses de recherche. Les extraits du recueil poétique Prière aux ancêtres de même que leur traduction illustrent la rencontre entre des univers différents notamment la culture tégué, la langue française et la langue italienne. Cette étude de cas nous a offert la possibilité de dépasser les frontières culturelles et linguistiques, en écoutant le texte, tout en tâchant de rendre justice au texte source lors d'un transfert interlingual. Nous envisageons ainsi un modèle transculturel qui respecte la diversité des langues et des cultures. Pour abattre les frontières linguistiques et culturelles, il faut réfléchir sur l'importance de l'hospitalité et de l'acceptation de l'autre, en 


\section{sciendo}

soulignant la valeur de la biographie linguistique des auteurs vu le caractère plurilingue du corpus en question. Il est donc impossible de penser la traduction comme un acte uniquement linguistique, car derrière les langues se cachent des choix, des mondes, des univers et des sens qui sont mobilisés dans les textes. Comme nous avons vu dans le cas de la traduction prise en examen dans notre étude, il est indispensable de connaitre (i) les dynamiques culturelles des textes et (ii) les références culturelles comme dans le cas de Mwènè et panthère (cf. 3.1). Toutefois, il faut accepter la perte sémantique, car dans la traduction à la fois on perd et on gagne des éléments, mais toujours dans ce rapport réciproque d'échange et de dynamique transculturelle, ce qui charge la traduction du pouvoir d'abattre les frontières culturelles. Dans le cadre spécifique de notre étude, par le biais de l'acceptation du monde culturel et linguistique du poète en italien, l'on pourrait penser dépasser les frontières linguistiques et culturelles en traduction et de bâtir une première étape pour la construction d'une approche transculturelle de la traduction, car la traduction fait partie d'un processus visant au dépassement des frontières linguistiques et culturelles. Nous concluons cette étude avec une réflexion d'Achille Mbembe :

« La frontière n'est plus seulement une ligne de démarcation qui sépare des entités souveraines distinctes. Dispositif ontologique, elle fonctionne désormais d'elle-même et en elle-même, anonyme et impersonnelle, avec ses lois propres. [...] Tout, en effet, nous ramène à la frontière, ce lieu zéro de la non-relation et du déni de l'idée même d'une humanité commune, d'une planète, la seule que nous aurions, qu'ensemble nous partagerions, et à laquelle nous lierait notre condition commune de passants. » (Mbembe, 2020 : 67-68)

\section{Références bibliographiques}

AMILHAT SZARY, Anne-Laure. Qu'est-ce qu'une frontière aujourd'hui ?. Paris : Presses Universitaires de France, 2015.

ANDERSON, Benedict. Imagined Communities Reflections on the Origin AND Spread of Nationalism, Revised ed. London New York: Verso, 1991.

APTER, Emily.Zones de traduction pour une nouvelle littérature comparée (Ouvertures). Paris: Fayard, 2015.

CASINI, Federica. "La poetica dell'ospitalità di Antonio Prete. Un traduttore in dialogo». In: Cahiers d'études romanes, 29 | 2014 : 245-257.

CHEVRIER, Jacques. Gabriel Okoundji, poète des deux fleuves. Ciboure : La Cheminante, 2014.

MBEMBE, Joseph-Achille. Brutalisme. Paris :la Découverte, 2020.

MIANO, Léonora. Habiter la frontière. Paris : L'Arche, 2012.

Meschonnic, Henri. Poétique du traduire. Paris : Verdier, 1999.

OKOUNDJI, Gabriel. Prière aux ancêtres. Gardonne: Fédérop, 2007.

OKOUNDJI, Gabriel. Au matin de la parole. Gardonne : Fédérop, 2009. 
RAGUET, Christine. «Y a-t-il des limites à la traduction transculturelle ?». In : Wecksteen, Corinne (dir.) ; El Kaladi, Ahmed (dir.). La traductologie dans tous ses états. Arras: Artois Presses Université, 2007: 39-54.

PRETE, Antonio. All'ombra dell'altra lingua. Per una poetica della traduzione. Torino: Bollati Boringhieri, 2011.

SEMUJIANGA, Josias. «Transculturalité». In : Vocabulaire des études francophones. Les concepts de base, Beniamino Michel, Gauvin Lise (dir.). Limoges : PULIM, $2005:$ 179-180.

TARDIVEL, Émilie. « Qu'est-ce qu'une nation ? ». Études (10|2016) : 53-64.

\section{Dictionnaire en ligne}

Trésor de la langue française informatisé. Disponible en ligne. URL: TLFi (atilf.fr) 